\title{
Tissue CA125 and HE4 Gene Expression Levels Offer Superior Accuracy in Discriminating Benign from Malignant Pelvic Masses
}

\author{
Amal Fawzy ${ }^{1}$, Mohamed R Mohamed ${ }^{2}$, Mohamed AM Ali²*, Mohamed H Abd \\ El-Magied ${ }^{1}$, Amany M Helal ${ }^{3}$
}

\begin{abstract}
Background: Ovarian cancer remains a major worldwide health care issue due to the lack of satisfactory diagnostic methods for early detection of the disease. Prior studies on the role of serum cancer antigen 125 (CA125) and human epididymis protein 4 (HE4) in detecting ovarian cancer presented conflicting results. New tools to improve the accuracy of identifying malignancy are urgently needed. We here aimed to evaluate the diagnostic utility of tissue CA125 and HE4 gene expression in comparison to serum CA125 and HE4 in discriminating benign from malignant pelvic masses. Materials and Methods: One-hundred Egyptian women were enrolled in this study, including 60 epithelial ovarian cancer (EOC) patients and 20 benign ovarian tumor patients, as well as 20 apparently healthy women. Preoperative serum levels of CA125 and HE4 were measured by immunoassays. Tissue expression levels of genes encoding CA125 and HE4 were determined by quantitative real time polymerase chain reaction (qRT-PCR). The diagnostic performance of CA125 and HE4, measured either as mRNA or protein levels, was evaluated by receiver operating characteristic (ROC) curves. Results: The serum CA125+HE4 combination and serum HE4, with area under the curve (AUC) values of 0.935 and 0.932 , respectively, performed significantly better than serum CA125 (AUC=0.592; $P<0.001$ ). Tissue $C A 125$ and HE4 (AUC=1) performed significantly better than serum CA125 $(P<0.001)$, serum HE4 $(P=0.016)$ and the serum CA125+HE4 combination $(\mathrm{P}=\mathbf{0 . 0 1 8})$. Conclusions: Measurement of tissue $\mathrm{CA125}$ and $\mathrm{HE} 4$ gene expression not only improves discriminatory performance, but also broadens the range of differential diagnostic possibilities in distinguishing EOC from benign ovarian tumors.
\end{abstract}

Keywords: Pelvic mass - differential diagnosis - cancer antigen 125 - human epididymis protein 4

Asian Pac J Cancer Prev, 17 (1), 323-333

\section{Introduction}

Ovarian cancer is the deadliest gynecological malignancy (Kobayashi et al., 2012). It is the seventh most frequent cancer and the seventh most common cause of cancer death among women worldwide. In Egypt, ovarian cancer represents the fifth most frequent cancer and the fifth most common cause of death from cancer in women (Ferlay et al., 2013). The poor prognosis of ovarian cancer is attributed to the fact that the cancer is insidious; generally asymptomatic in the early stages and initial symptoms occur only during the late stage of the disease, so that approximately $75 \%$ of ovarian cancer cases present at a late stage (Berek et al., 2012).

So far, cancer antigen 125 (CA125) is the most widely used serum biomarker for detecting and monitoring ovarian cancer in the clinical setting (Bast et al., 2005; Duffy et al., 2005; Sturgeon et al., 2008; Medeiros et al., 2009). Nevertheless, elevated serum CA125 levels are only seen in $50 \%$ of patients presented with early stage ovarian cancer and $80 \%-90 \%$ of patients with late stage disease, thus limiting its sensitivity (Molina et al., 1992; Rosen et al., 2005). Furthermore, the utility of CA125 as a biomarker for detecting ovarian cancer is hindered by the documented lack of specificity, since elevated serum CA125 concentrations can be found not only in a broad range of benign gynecologic diseases, but also in malignancies of different origin, including non-ovarian gynecologic cancers, other epithelial tumors and nonepithelial malignancies (Buamah, 2000; Miralles et al., 2003; Somigliana et al., 2004; Molina et al., 2008; Park et al., 2011).

The poor sensitivity and specificity of CA125 have stimulated the search for a more reliable biomarker sought to improve the diagnostic accuracy of identifying malignancy in women with a pelvic mass (Havrilesky et al., 2008). Among a wide spectrum of serum biomarkers proposed to aid in the diagnosis of women presenting 
with a suspicious pelvic mass, human epididymis protein 4 (HE4), has been introduced as a novel biomarker for ovarian cancer diagnosis (Hellström et al., 2003; Su et al., 2013). HE4 was found to offer a better diagnostic performance than CA125 for distinguishing between benign and malignant pelvic masses (Holcomb et al., 2011; Zheng and Gao, 2012; Chung et al., 2013). Regardless of its beneficial characteristics, similar to classic tumor markers (Trapé et al., 2011), HE4 has some limitations in the detection of tumors, where its level may be elevated in benign diseases without evidence of malignancy (Hertlein et al., 2012).

Prior studies on the role of CA125 and HE4 in the differential diagnosis of ovarian masses reported controversial and inconsistent conclusions. Although several meta-analyses have reported that HE4 had a better performance than CA125 for the diagnosis of ovarian cancer (Yu et al., 2012; Ferraro et al., 2013; Yang et al., 2013; Zhen et al., 2104), in other studies (Montagnana et al., 2009; Partheen et al., 2011; Van Gorp et al., 2011), it has been indicated that HE4 is not superior to CA125 in differentiating benign ovarian masses from malignant ovarian tumors.

Several reports have proposed that combining the measurements of serum CA125 and HE4 levels yielded a higher accuracy compared to either biomarker alone for the differential diagnosis of pelvic masses (Moore et al., 2008; Huhtinen et al., 2009; Moore et al., 2009; Escudero et al., 2011; Lenhard et al., 2011; Freydanck et al., 2012). However, it has been shown that no benefit from combining CA125 and HE4 in the clinical practice (Jacob et al., 2011).

An algorithm for risk scoring called the risk of ovarian malignancy algorithm (ROMA) based on menopausal status and preoperative measurement of serum CA125 and HE4 levels has been proposed for stratifying women with pelvic mass scheduled to undergo surgery into lowand high-risk groups (Moore et al., 2009). Although several studies have reported that ROMA yielded a better diagnostic accuracy than either marker alone (Moore et al., 2008; Moore et al., 2009; Bandiera et al., 2011; Molina et al., 2011; Moore et al., 2011; Li et al., 2012; Ortiz-Muñoz et al., 2014; Fujiwara et al., 2015), other studies failed to demonstrate its superiority over CA125 or HE4 alone for assessing the risk of ovarian cancer (Montagnana et al., 2011; Van Gorp et al., 2011; Wang et al., 2014).

Data from previous studies on the accuracy of serum CA125 and HE4 as well as ROMA in ovarian cancer diagnosis are contradictory and it has not been conclusively determined which of these markers yields the best performance. With all of this in mind, there is a pressing need to identify new tools with higher diagnostic accuracy for ovarian cancer diagnosis. In view of the clinical importance of diagnosing ovarian cancer with a better performance and in an attempt to identify a more accurate diagnostic tool, the current study was conducted to evaluate the diagnostic accuracy of tissue CA125 and HE4 gene expression levels in comparison to serum CA125 and HE4 levels as well as ROMA in a cohort of Egyptian women with a pelvic mass.

\section{Materials and Methods}

\section{Study population and design}

Eighty patients who were diagnosed with a pelvic mass suspected for ovarian cancer and were scheduled for surgical intervention at the Surgery Department, National Cancer Institute, Cairo University, Cairo, Egypt from October 2012 to August 2013 were prospectively enrolled in this case-control study. In addition, 20 agematched healthy women who admitted to the Surgery Department, National Cancer Institute, Cairo University, Cairo, Egypt for physical examination and had no medical history of malignant diseases were recruited as controls. To be eligible for enrollment, patients were required to be 18 years of age or older and have a diagnosis of a pelvic mass. All patients underwent radiologic imaging prior to surgery to document the presence of a pelvic mass.

All patients underwent surgical removal of the ovarian mass. Tissue specimens were obtained from all patients and reviewed by three experienced gynecological pathologists to verify the diagnoses made by the site pathologists as either benign or malignant. Twenty normal ovarian specimens were obtained from apparently normal tissue excised from the safety margins of the patients. Histological type and differentiation grade of ovarian cancer tissues were determined according to World Health Organization (WHO) criteria. Ovarian cancer surgical staging was performed according to the International Federation of Gynecology and Obstetrics (FIGO) guidelines. Early stage was defined as FIGO stages I \& II, while late stage was defined as FIGO stages III \& IV. Patients' demographic and clinical data were retrieved from the patients' medical records. Women were considered to be postmenopausal if they had not experienced a menstrual period for at least one year prior to enrollment, or if they were $>55$ years old and the date of the last menstrual period was unknown. Women were considered to be premenopausal if they had a period within 1 year of the study entry or if they were $<48$ years old and the date of their last menstrual period was unknown. Exclusion criteria include: 1) Pregnancy, 2) Chronic liver disease, 3) Chronic renal failure. All enrolled patients and healthy women provided written informed consent prior to inclusion into the study in accordance with the Declaration of Helsinki. The study protocol was approved by the scientific ethical committee of National Cancer Institute, Cairo University, Cairo, Egypt.

\section{Measurement of serum CA125 and HE4 levels}

Blood samples were collected from patients and healthy women into plastic BD vacutainer rapid serum tubes (Becton, Dickinson and Company, Franklin Lakes, NJ, USA) by venipuncture. The blood samples were obtained from the patients on the day preceding the planned surgery. Sera were immediately obtained, aliquoted in sterile polypropylene tubes and stored at $-80^{\circ} \mathrm{C}$ until analysis was carried out. Preoperative serum levels of CA125 and HE4 were measured using the Architect CA125II chemiluminescent microparticle immunoassay (Abbott Diagnostics, Abbott Park, IL, USA) and the HE4 enzyme immunoassay (EIA; Fujirebio 
Tissue CA125 and HE4 Gene Expression Offers Superior Accuracy in Discriminating Benign from Malignant Pelvic Masses

Diagnostics, Malvern, PA, USA), respectively, according to the manufacturer's instructions.

\section{Calculation of the ROMA score}

The ROMA score combines CA125 and HE4 values along with the menopausal status into a predictive index $(\mathrm{PI})$, which in turn is used to calculate the predicted probability (PP) of ovarian cancer (from 0 to $100 \%$ ). ROMA score was calculated according to the previously described formulae (Moore et al., 2009; Moore et al., 2010), using logistic regression analysis for premenopausal and postmenopausal women as follows, where LN is the natural logarithm: Premenopausal women $\mathrm{PI}=-12.0+2$ $.38 \times$ LN[HE4]+0.0626 $\times$ LN[CA125]; postmenopausal women PI=-8.09+1.04 $\times$ LN[HE4]+0.732 $\times$ LN[CA125]; $\mathrm{PP}=\exp (\mathrm{PI}) /[1+\exp (\mathrm{PI})]$; and ROMA $(\%)=\exp (\mathrm{PI}) /$ $[1+\exp (\mathrm{PI})] \times 100$.

\section{Quantification of tissue CA125 and HE4 mRNA levels}

The gene expression levels of mucin 16 (MUC16) and whey acidic protein (WAP) four-disulfide core domain 2 (WFDC2), which encode the CA125 and HE4 proteins, respectively, were quantified in normal, benign and malignant tissues by quantitative real time polymerase chain reaction (qRT-PCR). Briefly, the tissue specimens were collected during surgery, snap-frozen and stored in liquid nitrogen until analysis was performed. Total RNA was purified using RNeasy Mini Kit (Qiagen, Valencia, CA, USA) according to the manufacturer's instructions. Single-stranded RNA was reverse transcribed into single-stranded complementary DNA (cDNA) using High-Capacity cDNA Reverse Transcription kit (Applied Biosystems, Foster City, CA, USA) following the manufacturer's instructions. Quantitative assessment of gene expression levels was performed by TaqMan gene expression assay (Applied Biosystems, Foster City, CA, USA) as recommended by the manufacturer. The StepOne Real-Time PCR System (Applied Biosystems, Foster City, CA, USA) was used for real-time analysis. Relative expression of MUC16 and WFDC2 genes was analyzed by the comparative $\mathrm{Ct}$ method $\left(2^{-\Delta \Delta \mathrm{Ct}}\right)$ (Livak and Schmittgen, 2001), using glyceraldehyde-3-phosphate dehydrogenase (GAPDH) as the endogenous control. Data were expressed as the fold change in MUC16 and WFDC2 gene expression in the patients normalized to the expression levels of the endogenous control and relative to the healthy controls.

\section{Statistical analysis}

Categorical variables were presented as the number of cases (percentage) and compared using the Pearson's chi-square $(\chi 2)$ test. The continuous variables were reported as median (interquartile range, IQR: 25 th quartile to 75th quartile) and the non-parametric Mann-Whitney $\mathrm{U}$ test and Kruskal-Wallis test were used to compare median levels of CA125 and HE4 between two and more than two groups, respectively. A 2-sided probability (P) value was used for all statistical analyses, and a $\mathrm{P}$ value of $<0.05$ was considered statistically significant. The correlation between each biomarker, measured either by immunoassays or by qRT-PCR, was analyzed using Spearman's rank correlation coefficient. To evaluate the diagnostic accuracy of CA125 and HE4, measured either as mRNA or protein levels, a receiver operating characteristic (ROC) curve was constructed and the areas under the curve (AUC) were calculated with $95 \%$ confidence intervals and compared using the nonparametric method developed by DeLong et al. (1988). Data statistical analyses were

\section{Table 1. Demographic and Clinical Characteristics of Epithelial Ovarian Cancer Patients}

\begin{tabular}{|c|c|c|c|c|}
\hline \multirow[t]{2}{*}{ Feature } & \multicolumn{3}{|c|}{ Epithelial ovarian cancer patients } & \multirow[b]{2}{*}{ P value } \\
\hline & $\begin{array}{c}\text { Premenopausal } \\
\mathrm{n}=34(56.7 \%)\end{array}$ & $\begin{array}{c}\text { Postmenopausal } \\
\mathrm{n}=26(43.3 \%)\end{array}$ & $\begin{array}{l}\text { Total } \\
n=60\end{array}$ & \\
\hline Age (years) & $\begin{array}{r}38.9 \pm 7.1 \\
(24-49)\end{array}$ & $\begin{array}{r}62.4 \pm 6.3 \\
(50-76)\end{array}$ & $\begin{array}{r}49.1 \pm 13.5 \\
(24-76)\end{array}$ & $<0.001 *$ \\
\hline \multicolumn{5}{|c|}{ Histological type } \\
\hline Serous & $21(61.8 \%)$ & $14(53.9 \%)$ & $35(58.3 \%)$ & $0.459 \dagger$ \\
\hline Endometrioid & $11(32.3 \%)$ & $11(42.3 \%)$ & $22(36.7 \%)$ & $0.660 \ddagger$ \\
\hline Mucinous & $2(5.9 \%)$ & $1(3.8 \%)$ & $3(5.0 \%)$ & $0.531 \S$ \\
\hline \multicolumn{5}{|c|}{ Differentiation grade } \\
\hline Grade 1 & $10(29.4 \%)$ & $9(34.6 \%)$ & $19(31.7 \%)$ & \\
\hline Grade 2 & $17(50.0 \%)$ & $8(30.8 \%)$ & $25(41.7 \%)$ & 1 vs. $3=0.600$ \\
\hline $\begin{array}{l}\text { Grade } 3 \\
\text { FIGO stage }\end{array}$ & $7(20.6 \%)$ & $9(34.6 \%)$ & $16(26.6 \%)$ & 2 vs. $3=0.124$ \\
\hline Stage I & $17(50.0 \%)$ & $7(26.9 \%)$ & $24(40.0 \%)$ & $\begin{array}{l}\text { I vs. } I I=0.001^{*} \\
\text { I vs. III }=0.679 \\
\text { I vs. } I V=1.000\end{array}$ \\
\hline Stage II & $2(5.9 \%)$ & $11(42.3 \%)$ & $13(21.7 \%)$ & II vs. III $=0.171$ \\
\hline Stage III & $13(38.2 \%)$ & $7(26.9 \%)$ & $20(33.3 \%)$ & II vs. IV $=0.136$ \\
\hline Stage IV & $2(5.9 \%)$ & $1(3.8 \%)$ & $3(5.0 \%)$ & III vs. IV $=1.000$ \\
\hline
\end{tabular}

FIGO, international federation of gynecology and obstetrics. Qualitative data are represented as the number of cases (\%), whereas quantitative data are represented as mean $\pm \mathrm{SD}$ (range, minimum-maximum). $\dagger$, Serous vs. Endometrioid; $\uparrow$, Serous vs. Mucinous; $\S$, Endometrioid vs. Mucinous. $*$ indicates a statistically significant difference. 
Amal Fawzy et al

performed using the statistical package for the social sciences (SPSS Statistics for Windows, Version 20.0; IBM Corp., Armonk, NY, USA).

\section{Results}

Demographic and clinical characteristics of the study cohort

The study cohort comprised of 100 women, with a mean age of 46.9 13.0 years (range 23-76 years), including 63 premenopausal women and 37 postmenopausal women. The participants were divided into 3 groups: 1) twenty healthy women with a mean age of $41.5 \pm 12.0$ years (range 23-62 years), including 15 premenopausal women and 5 postmenopausal women; 2) twenty benign ovarian tumor women with a mean age of $45.8 \pm 11.0$ years (range 25-65 years), including 14 premenopausal women and 6 postmenopausal women; 3 ) sixty epithelial ovarian cancer (EOC) women. Of the 20 benign ovarian tumor patients, 8 patients had adenofibroma ( 5 premenopausal women and 3 postmenopausal women), 8 patients had serous cystadenoma ( 6 premenopausal women and 2 postmenopausal women) and 4 patients had mucinous cystadenoma ( 3 premenopausal women and 1 postmenopausal women). The demographic and clinical features of EOC patients are summarized in Table 1.

Serum CA125 and HE4 concentrations and ROMA score

The median concentration of CA125 in benign ovarian tumor patients and those with EOC was significantly higher than that in healthy controls $(\mathrm{P}<0.05)$. Although the median CA125 serum concentration in EOC patients was higher than that in benign ovarian tumor patients, this difference did not reach statistical significance $(\mathrm{P}=0.222)$. The median serum HE4 level in EOC patients was significantly higher than that in benign ovarian tumor patients and in healthy controls $(\mathrm{P}<0.001)$, whereas the concentrations of HE4 in benign ovarian tumor patients

Table 2. Serum CA125 and HE4 Concentrations and ROMA Score among Healthy women, benign ovarian tumor patients and Epithelial Ovarian Cancer Patients

\begin{tabular}{|c|c|c|c|c|}
\hline \multirow[t]{2}{*}{ Biomarker } & \multicolumn{3}{|c|}{ Participants $(n=100)$} & \multirow[t]{2}{*}{$\mathrm{P}$ value } \\
\hline & $\begin{array}{l}\text { Healthy women } \\
\qquad(n=20)\end{array}$ & $\begin{array}{l}\text { Benign ovarian tumors } \\
\qquad(\mathrm{n}=20)\end{array}$ & $\begin{array}{l}\text { Epithelial ovarian cancer } \\
\qquad(\mathrm{n}=60)\end{array}$ & \\
\hline \multicolumn{5}{|l|}{ CA125 (U/mL) } \\
\hline Premenopausal & $4.7(0.7-8.9)$ & $12.4(5.9-16.8)$ & $11.6(3.1-55.0)$ & $\begin{array}{l}0.014^{\dagger *} \\
0.013^{\ddagger *} \\
0.525^{\S}\end{array}$ \\
\hline Postmenopausal & $1.6(0.7-5.7)$ & $12.6(6.3-16.1)$ & $14.5(5.3-191.7)$ & $\begin{array}{l}0.082^{\dagger} \\
0.011^{\ddagger} * \\
0.381^{\S}\end{array}$ \\
\hline Total & $4.7(0.8-6.9)$ & $12.4(6.7-16.5)$ & $13.7(3.7-113.4)$ & $\begin{array}{c}0.001^{\dagger *} \\
<0.001^{\ddagger *} \\
0.222^{\S}\end{array}$ \\
\hline $\begin{array}{l}\text { P value } \\
\text { HE4 (pmol/L) }\end{array}$ & $0.672^{\Delta}$ & $0.968^{\sharp}$ & $0.318^{¥}$ & \\
\hline Premenopausal & $32.2(30.0-41.1)$ & $34.4(32.0-36.1)$ & $109.4(49.9-477.2)$ & $\begin{array}{c}0.310^{\dagger} \\
<0.001^{\text {} *} \\
<0.001^{\S *}\end{array}$ \\
\hline Postmenopausal & $35.0(30.3-45.1)$ & $35.1(30.9-45.0)$ & $84.7(58.9-245.9)$ & $\begin{array}{c}0.931^{\dagger} \\
<0.001^{\ddagger *} \\
<0.001^{\text {} *}\end{array}$ \\
\hline Total & $32.7(30.5-41.5)$ & $34.4(32.4-36.6)$ & $93.8(54.8-412.6)$ & $\begin{array}{c}0.369^{\dagger} \\
<0.001^{\ddagger *} \\
<0.001^{\S *}\end{array}$ \\
\hline $\begin{array}{l}\mathrm{P} \text { value } \\
\text { ROMA score }(\%)\end{array}$ & $0.306^{\Delta}$ & $0.718^{\mathfrak{J}}$ & $0.881^{¥}$ & \\
\hline Premenopausal & $2.7(2.2-3.7)$ & $3.0(2.6-3.5)$ & $31.5(7.7-94.3)$ & $\begin{array}{c}0.310^{\dagger} \\
<0.001^{\text {} *} \\
<0.001^{\text {} *}\end{array}$ \\
\hline Postmenopausal & $2.2(1.0-4.2)$ & $8.0(4.1-9.5)$ & $20.8(9.5-55.6)$ & $\begin{array}{c}0.082^{\dagger} \\
<0.001^{\ddagger *} \\
0.008^{\S} *\end{array}$ \\
\hline Total & $2.7(2.1-3.8)$ & $3.2(2.6-6.4)$ & $29.6(8.9-76.8)$ & $\begin{array}{r}0.049^{\ddagger *} \\
<0.001^{\ddagger *} \\
<0.001^{\text {} *}\end{array}$ \\
\hline & $P$ value & $0.612^{\Delta}$ & $0.026^{9 *}$ & $0.586^{¥}$ \\
\hline
\end{tabular}

CA125, cancer antigen 125; HE4, human epididymis protein 4; ROMA, risk ovarian malignancy algorithm. Data are represented as median (interquartile range, IQR: 25 th quartile to 75 th quartile). $\dagger$, Healthy women vs. Benign ovarian tumors; $\$$, Healthy women vs. Epithelial ovarian cancer; §, Benign ovarian tumors vs. Epithelial ovarian cancer. $\Delta$, I and $¥$, the difference in the CA125 and HE4 levels as well as ROMA score between pre- and post-menopausal women in Healthy women, Benign ovarian tumors and Epithelial ovarian cancer groups, respectively. $*$ indicates a statistically significant difference 
Tissue CA125 and HE4 Gene Expression Offers Superior Accuracy in Discriminating Benign from Malignant Pelvic Masses

were equivalent to those in healthy controls $(\mathrm{P}=0.369)$. The median ROMA scores differed significantly among healthy controls, benign ovarian tumor patients and EOC patients $(\mathrm{P}<0.05)($ Table 2$)$.

Table 3 shows the CA125 and HE4 serum levels as well as ROMA scores in EOC patients, subdivided according to histological type, differentiation grade and FIGO stage. Among EOC patients, the median CA125 concentration and ROMA score did not differ significantly among patients with endometrial, serous and mucinous adenocarcinoma $(\mathrm{P}>0.05)$. On the other hand, the median HE4 concentration was significantly higher in patients with serous adenocarcinoma than that in patients with endometrial adenocarcinoma $(\mathrm{P}=0.029)$, whereas the median HE4 concentration did not differ significantly neither between patients with serous and mucinous adenocarcinoma nor between patients with endometrial and mucinous adenocarcinoma $(\mathrm{P}>0.05)$. There were no statistically significant differences in the median CA125 and HE4 levels as well as ROMA scores among EOC patients with the different differentiation grades $(\mathrm{P}>0.05)$. Serum CA125 and HE4 levels as well as ROMA scores were found to be related to FIGO stage with higher values in late stages than in early stages, but this difference did not reach statistical significance $(\mathrm{P}>0.05)$.

Tissue expression of CA125 and HE4 encoding genes The fold change in the expression levels of MUC16

Table 3. CA125 and HE4 Serum Concentrations and ROMA Score in Epithelial Ovarian Cancer Patients According to histological type, differentiation grade and FIGO Stage

\begin{tabular}{|c|c|c|c|c|c|c|}
\hline Variable & CA125 (U/mL) & $P$ value & HE4 (pmol/L) & P value & ROMA score $(\%)$ & $\mathrm{P}$ value \\
\hline \multicolumn{7}{|l|}{ Histological type } \\
\hline \multirow[t]{2}{*}{ Serous $(n=35)$} & $\begin{array}{c}12.1 \\
(1.7-36.5)\end{array}$ & & $\begin{array}{c}149.1 \\
(54.3-493.9)\end{array}$ & & $\begin{array}{c}28.8 \\
(8.8-94.4)\end{array}$ & \\
\hline & & $\begin{array}{l}0.119 \dagger \\
0.294 \$\end{array}$ & & $\begin{array}{l}0.029 \dagger * \\
0.720 \ddagger\end{array}$ & & $\begin{array}{l}0.317 \dagger \\
0.346 \$\end{array}$ \\
\hline Endometrioid $(\mathrm{n}=22)$ & $\begin{array}{c}34.2 \\
(6.1-131.8)\end{array}$ & $0.616 \S$ & $\begin{array}{c}74 \\
(50.8-106.2)\end{array}$ & $0.112 \S$ & $\begin{array}{c}23 \\
(7.7-51.6)\end{array}$ & $0.112 \S$ \\
\hline Mucinous ( $n=3$ ) & $\begin{array}{c}36.7 \\
(8.8-290.4)\end{array}$ & & $\begin{array}{c}173.5 \\
(69.8-807.3)\end{array}$ & & $\begin{array}{c}60 \\
(26.2-98.6)\end{array}$ & \\
\hline \multicolumn{7}{|l|}{ Differentiation grade } \\
\hline Grade $1(n=19)$ & $\begin{array}{c}12.9 \\
(1.4-36.5)\end{array}$ & & $\begin{array}{c}149.1 \\
(54.3-355.1)\end{array}$ & & $\begin{array}{c}34.8 \\
(8.8-77.0)\end{array}$ & \\
\hline Grade $2(n=25)$ & $\begin{array}{c}13.6 \\
(5.0-144.9)\end{array}$ & $\begin{array}{l}1 \text { vs. } 2=0.337 \\
1 \text { vs. } 3=0.243 \\
2 \text { vs. } 3=0.843\end{array}$ & $\begin{array}{c}89.3 \\
(56.8-333.6)\end{array}$ & $\begin{array}{l}1 \text { vs. } 2=0.470 \\
1 \text { vs. } 3=0.461 \\
2 \text { vs. } 3=0.534\end{array}$ & $\begin{array}{c}31.3 \\
(8.0-79.0)\end{array}$ & $\begin{array}{l}1 \text { vs. } 2=0.924 \\
1 \text { vs. } 3=1.000 \\
2 \text { vs. } 3=0.905\end{array}$ \\
\hline Grade $3(n=16)$ & $\begin{array}{c}23.4 \\
(4.6-165.8)\end{array}$ & & $\begin{array}{c}71.3 \\
(51.0-438.8)\end{array}$ & & $\begin{array}{c}18.5 \\
(10.9-84.8)\end{array}$ & \\
\hline \multicolumn{7}{|l|}{ FIGO stage } \\
\hline Early stage $(\mathrm{I}+\mathrm{II})(\mathrm{n}=37)$ & $\begin{array}{c}12.9 \\
(2.7-114.2)\end{array}$ & & $\begin{array}{c}81.7 \\
(56.0-173.4)\end{array}$ & & $\begin{array}{c}21.5 \\
(9.7-60.0)\end{array}$ & \\
\hline Late stage $(\mathrm{III}+\mathrm{IV})(\mathrm{n}=23)$ & 3) $\begin{array}{c}14 \\
(5.5-114.5)\end{array}$ & 0.589 & $\begin{array}{c}103 \\
(53.2-453.9)\end{array}$ & 0.489 & $\begin{array}{c}34.8 \\
(8.7-92.9)\end{array}$ & 0.676 \\
\hline
\end{tabular}

CA125, cancer antigen 125; HE4, human epididymis protein 4; ROMA, risk ovarian malignancy algorithm. Data are represented as median (interquartile range, IQR: 25 th quartile to 75 th quartile or range, minimum-maximum as appropriate). $\dagger$, Serous vs. Endometrioid; $\ddagger$, Serous vs. Mucinous; $\S$, Endometrioid vs. Mucinous. * indicates a statistically significant difference

Table 4. Expression Levels Fold Change of MUC16 (Encoding CA125) and WFDC2 (encoding HE4) in Tissue Specimens of Patients with Benign Ovarian Tumor and EOC Relative to Healthy Controls

\begin{tabular}{|c|c|c|c|c|}
\hline \multirow[t]{2}{*}{ Study group } & \multicolumn{2}{|c|}{ MUC16 (CA125) } & \multicolumn{2}{|c|}{ WFDC2 (HE4) } \\
\hline & Fold change & $\mathrm{P}$ value & Fold change & $\mathrm{P}$ value \\
\hline \multicolumn{5}{|c|}{ Benign ovarian tumors $(n=20)$} \\
\hline Premenopausal $(n=14)$ & $1.26(1.18-1.34)$ & $0.012 *$ & $1.22(1.11-1.59)$ & 0.968 \\
\hline Postmenopausal $(n=6)$ & $1.10(1.03-1.18)$ & & $1.31(1.13-1.54)$ & \\
\hline Total & $1.23(1.10-1.30)$ & & $1.24(1.11-1.52)$ & \\
\hline \multicolumn{5}{|c|}{ Epithelial ovarian cancer $(n=60)$} \\
\hline Premenopausal $(n=34)$ & $16.1(5.3-62.5)$ & 0.571 & $84.4(13.5-543.5)$ & 0.704 \\
\hline Postmenopausal $(n=26)$ & $14.0(6.0-31.1)$ & & $43.9(15.5-526.8)$ & \\
\hline Total & $14.9(6.1-48.5)$ & & $58.6(14.7-444.0)$ & \\
\hline & $<0.001 \dagger^{*}$ & & $<0.001 \dagger^{*}$ & \\
\hline
\end{tabular}

CA125, cancer antigen 125; HE4, human epididymis protein 4, MUC16, mucin 16; WFDC2, whey acidic protein (WAP) four-disulfide core domain 2. Fold change is the normalized MUC16 and WFDC2 expression in the benign ovarian tumors/Epithelial ovarian cancer group relative to the normalized MUC16 and WFDC2 expression in the healthy women group. Fold change is represented as median (interquartile range, IQR: 25th quartile to 75th quartile). $\dagger$, the difference in the fold change between Benign ovarian tumor and Epithelial ovarian cancer groups, independently from menopausal status and for pre- and post-menopausal women as separate groups. * indicates a statistically significant difference 


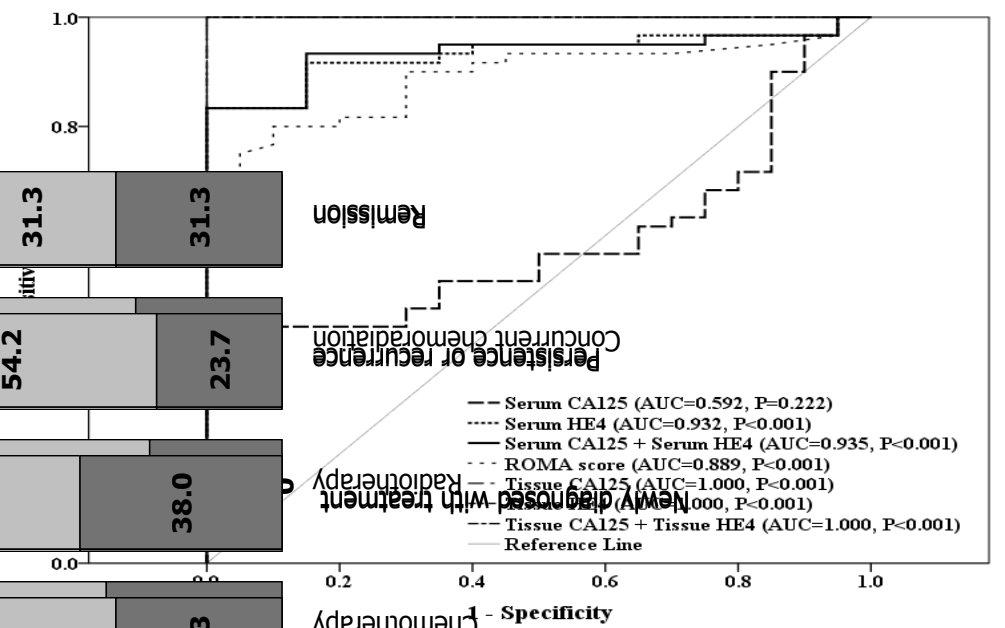

Figure 1. ROC Curve Analysis of Serum and Tissue CA125 and HE4, Either Alone or in Combination, and of ROMA for the Diagnosis of EOC by Comparing Benign Ovarian Tumor Patients with EOC Patients

and WFDC2 genes in ovarian tissue specimens of healthy controls, benign ovarian tumor patients and EOC patients is shown in Table 4. The median fold change of MUC16 and WFDC2 expression levels was significantly higher in EOC patients than that in benign ovarian tumor patients, for the whole group of patients $(\mathrm{P}<0.001)$ as well as for the pre- and postmenopausal patients separately $(\mathrm{P}<0.001)$.

\section{Correlation between CA125, HE4 and ROMA}

Correlation between CA125 and HE4, measured either as mRNA or protein levels, as well as ROMA score in benign ovarian tumor patients and those with EOC are presented in Table 5.

\section{Diagnostic accuracy of CA125, HE4 and ROMA}

The diagnostic performance of serum and tissue CA125 and HE4, either alone or in combination, as well as of ROMA was assessed by ROC curve analysis in the benign versus malignant cohorts (Table 6, Figure1). Serum CA125+serum HE4 combination and serum HE4 as well as ROMA with AUC values of $0.935,0.932$ and 0.889 , respectively, performed significantly better than serum CA125 alone $(\mathrm{AUC}=0.592$; $\mathrm{P}<0.001$ ). Additionally, serum HE4 and serum CA125+serum HE4 combination yielded similar diagnostic accuracy. Furthermore, serum CA125+serum HE4 combination and serum HE4 did not show better diagnostic performance than ROMA. On the other hand, tissue CA125 and HE4, either alone or in combination, displayed the same discriminatory performance with the highest AUC. Accordingly, tissue CA125 and HE4, either alone or in combination, performed significantly better than serum CA125 alone (0.592 vs $1 ; \mathrm{P}<0.001)$, serum HE4 alone (0.932 vs 1; $\mathrm{P}=0.016)$, serum CA125+serum HE4 combination (0.935 vs $1 ; \mathrm{P}=0.018)$ and ROMA (0.889 vs $1 ; \mathrm{P}=0.002)$.

\section{Discussion}

Ovarian cancer has a major impact on health care, being the second most common malignancy in women and the leading cause of death from gynecological malignancies. The clinical symptoms of ovarian cancer are vague and similar to those observed in other benign conditions; hence, the majority of patients are often diagnosed at a late stage which almost always translates into a poor prognosis (Seibaek et al., 2011). To date, there are a growing number of patients who present with a pelvic mass; therefore, it is necessary to identify the potential malignant ovarian disease 


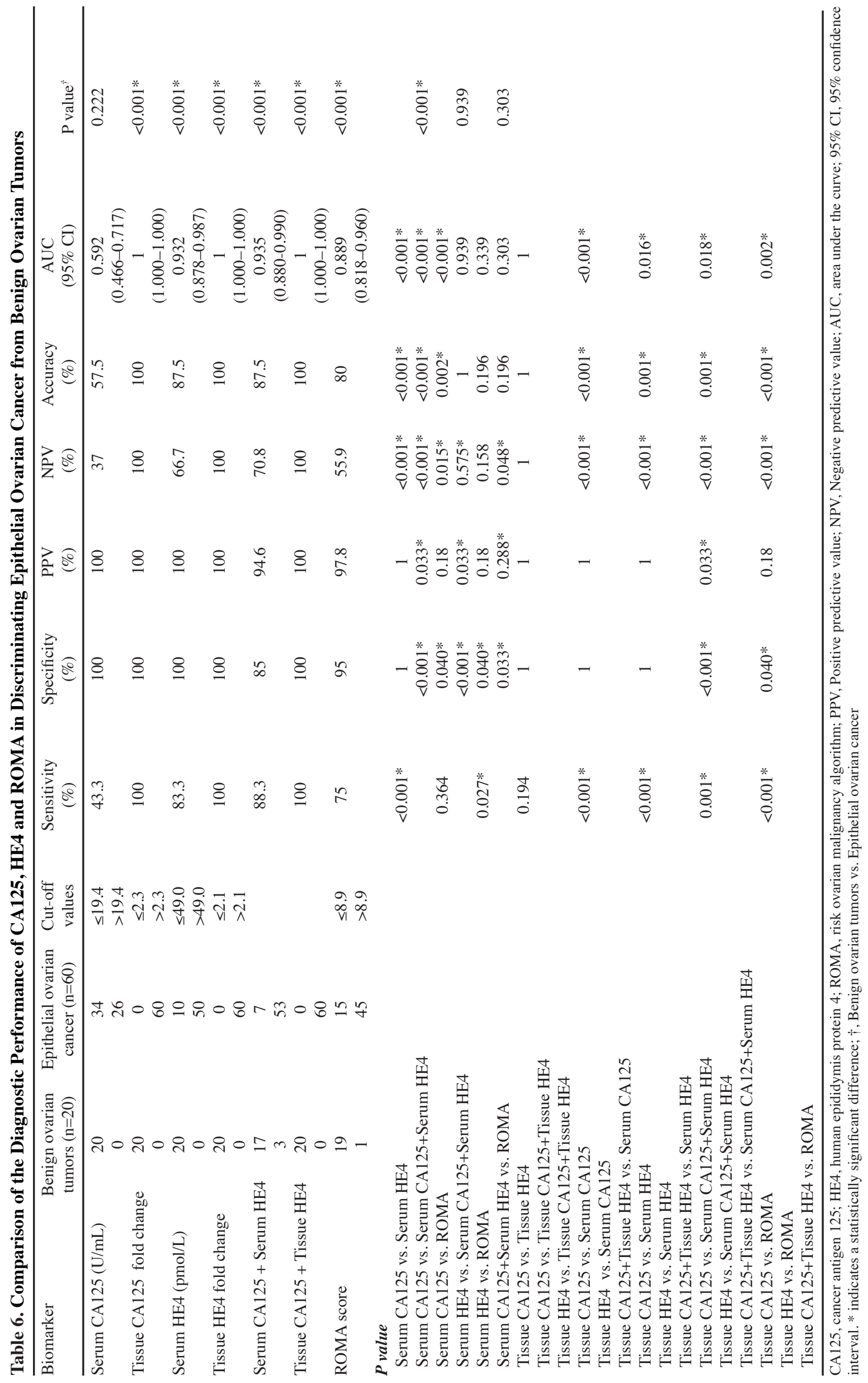


risk as early as possible to improve clinical outcome and survival of patients (Kobayashi et al., 2012).

Although several studies have been designed for examining possible associations between serum CA125 or HE4 measurements and ovarian cancer diagnosis, data from previous studies on the use of CA125 and HE4 in the differential diagnosis of ovarian cancer are inconsistent. There may be several explanations for the heterogeneity observed among previous studies, and in our opinion, the most intriguing are the differences in the study design, study population, demographic characteristics of patients, ovarian cancer prevalence, sample size, control group (healthy women or women with benign diseases), methods of measuring serum CA125 or HE4 levels (ELISA or chemiluminescent microparticle immunoassay) and cutoff values of CA125/HE4 assays (manufacturer or clinical practice). Furthermore, several studies did not take into account the effect of the uneven distribution of patients in pre- and post-menopausal status, histological subtypes and FIGO stages when interpreting the results of CA125 and HE4 measurements (Moore et al., 2009; Moore et al., 2010; Kim et al., 2011; Montagnana et al., 2011; Ruggeri et al., 2011; Van Gorp et al., 2011). Overall, each of these points theoretically represent a source of heterogeneity among studies likely influencing the markers' diagnostic performances.

The improvement of specificity without compromising sensitivity is essential for determining the risk of malignancy in patients presenting with pelvic mass. Although an inexpensive, sensitive and specific serum test would be the most attractive approach for diagnosing ovarian cancer, fundamental limitations of this approach have been recognized. In light of these observations, new tools for the diagnosis of ovarian cancer are urgently needed.

We investigated the clinical utility of tissue CA125 and HE4 gene expression in comparison to serum CA125 and HE4 in assessing the likelihood of malignancy in a cohort of Egyptian women with a pelvic mass.

In the current study, serum CA125 and HE4 levels as well as the ROMA scores differed among healthy controls, benign ovarian tumor women and EOC women. Our results are somewhat consistent with those of previous studies (Hellström et al., 2003; Moore et al., 2008; Huhtinen et al., 2009; Montagnana et al., 2009; Moore et al., 2009; Moore et al., 2010; Nolen et al., 2010; Bandiera et al., 2011; Molina et al., 2011; Park et al., 2011; Van Gorp et al., 2011; Karlsen et al., 2012; Park et al., 2012), which reported that serum CA125 and HE4 levels were significantly higher in ovarian cancer women than those in benign ovarian tumor women and in healthy women. In accordance with previous observations (Escudero et al., 2011), our results showed that serum CA125 and HE4 levels were found to be related to FIGO stage with higher concentrations in late stages (stage III/IV) than in early stages (stage I/II).

Our results showed that the fold change of MUC16 expression was significantly higher in EOC patients than that in benign ovarian tumor patients. Previous reports indicated that significant levels of CA125 were found in deposits of endometriosis and in some benign ovarian tumors (Meden and Fattahi-Meibodi, 1998; Fuith et al., 1987; Niloff et al., 1984).

Results of the current study revealed that the fold change of WFDC2 expression was significantly higher in EOC patients than that in benign ovarian tumor patients. Gene expression profiles indicated that WFDC2 is one of the most frequently upregulated genes in epithelial ovarian carcinomas with the highest expression being reported in serous ovarian carcinomas (Galgano et al., 2006). The mechanism of HE4 overexpression in ovarian cancer is not clear. However, it has been demonstrated that the chromosomal region where HE4 is located is frequently amplified in ovarian cancer and breast cancer (Berry et al., 2004).

Our data clearly demonstrated that serum HE4 displayed better diagnostic performance when compared with serum CA125 in differentiating EOC patients from those with benign ovarian tumors. These results support the hypothesis that HE4 could replace CA125 measurement as a standalone biomarker for ovarian cancer diagnosis.

Prior studies have reported conflicting results about the accuracy of serum CA125 and HE4 in the differential diagnosis of ovarian cancer. Several studies have demonstrated that HE4 had a better diagnostic performance than CA125 for discriminating ovarian cancer from benign gynecologic diseases (Moore et al., 2008; Huhtinen et al., 2009; Holcomb et al., 2011; OrtizMuñoz et al., 2014). A previous meta-analysis by Yu et al., (Yu et al., 2012) has reported that HE4 was found to be better than CA125 as an indicator for the diagnosis of ovarian cancer. In a subsequent systematic review, Ferraro et al., revised the available literature on the comparison between the diagnostic accuracy of HE4 and CA125. They suggested that HE4 measurement seems to be superior to CA125 in terms of diagnostic performance for the identification of ovarian cancer in women with suspected gynecological diseases (Ferraro et al., 2013). Furthermore, Yang et al., gathered all the evidence reported in literature on the clinical value of serum HE4 in the diagnosis of ovarian cancer. They demonstrated that the sensitivity and specificity of HE4 was higher than that of CA125 (Yang et al., 2013). Recently, Zhen et al., conducted a meta-analysis of the available evidence on the diagnostic accuracy of HE4 and CA125. They reported that HE4 appears to be superior to CA125 regarding diagnostic accuracy in distinguishing ovarian cancer from other benign gynecological diseases (Zhen et al., 2014). Conversely, in other studies (Park et al., 2011; Van Gorp et al., 2011; Li et al., 2012), it has been indicated that HE4 did not perform significantly better than CA125 in the diagnosis of ovarian cancer. Also, Li et al., (Li et al., 2012) performed a meta-analysis and demonstrated that HE4 is not superior to CA125 in predicting ovarian cancer.

Data of the present study showed that the combination of serum HE4 with serum CA125 displayed better diagnostic performance than serum CA125 alone, while revealed similar performance to that of serum HE4 in distinguishing ovarian cancer from benign ovarian tumors. These findings make it reasonable that the combination improved CA125 but not HE4 performance. 
Tissue CA125 and HE4 Gene Expression Offers Superior Accuracy in Discriminating Benign from Malignant Pelvic Masses

Several studies (Moore et al., 2008; Huhtinen et al., 2009; Moore et al., 2009; Escudero et al., 2011; Lenhard et al., 2011; Freydanck et al., 2012; Zhen et al., 2014) reported that the combination of serum HE4 with serum CA125 further enhanced the diagnostic accuracy when compared with either maker alone, suggesting that when used in combination, the two markers HE4 and CA125 complement each other, as each improves the discriminatory performance of the other. On the contrary, Jacob et al., suggested that no benefit from combining HE4 and CA125 in the clinical setting (Jacob et al., 2011).

In our study, although ROMA yielded a significantly better diagnostic performance than that of serum CA125 alone, ROMA did not perform significantly better than serum HE4 and serum HE4 plus serum CA125 combination for the differentiation between EOC patients and those with benign ovarian tumors.

Previous studies on the diagnostic performance of ROMA compared to either CA125 or HE4 in the differential diagnosis of ovarian cancer have provided conflicting results. Several reports have demonstrated that ROMA had a better diagnostic performance than either marker alone (Moore et al., 2008; Moore et al., 2009; Bandiera et al., 2011; Molina et al., 2011; Moore et al., 2011; Ortiz-Muñoz et al., 2014; Fujiwara et al., 2015). These findings appear to be in line with those of Li et al., (Li et al., 2012) who conducted a meta-analysis to clarify conflicting results existing in the diagnostic performance comparison among ROMA, HE4 and CA125. They suggested that ROMA was less specific but more sensitive than HE4, while both ROMA and HE4 were more specific than CA125 for EOC prediction. On contrary, a previous report (Montagnana et al., 2011) has demonstrated that ROMA did not show better diagnostic performance than HE4 alone in the diagnosis of ovarian cancer. Additionally, in another study (Van Gorp et al., 2011), it has been reported that combining HE4 and CA125 in the ROMA improved HE4 but not CA125 performance, indicating that ROMA and HE4 did not perform significantly better than CA125 alone. Recently, Wang et al., performed a meta-analysis to evaluate the diagnostic value of CA125, HE4 and ROMA for diagnosing ovarian cancer. They reported that the three markers had similar discriminatory performance in ovarian cancer diagnosis (Wang et al., 2014).

Here, we reported that tissue CA125 and HE4, either alone or in combination, perform significantly better than serum CA125 alone, serum HE4 alone and serum CA125+serum HE4 combination as well as ROMA in distinguishing EOC patients from benign ovarian tumor patients.

Although our results are promising, further prospective studies on a larger sample population are certainly necessary to reinforce this preliminary evidence and to assess the usefulness of measuring the expression levels of tissue CA125 and HE4 encoding genes more accurately before we can finally conclude that these measurements are of real clinical significance.

In conclusion, findings of the present study not only confirm the diagnostic value of serum CA125 and HE4 levels as well as ROMA already suggested by other reports, but also add a clinically relevant information, as for the first time we showed that tissue CA125 and HE4 gene expression demonstrates the highest discriminatory power in the differential diagnosis of pelvic masses, suggesting that measuring the expression levels of tissue CA125 and HE4 encoding genes, either alone or in combination, might provide a more accurate tool for distinguishing between EOC patients and those with benign ovarian tumors. Our observations support the notion that a diagnostic approach that accurately detect ovarian cancer would help planning patients' treatment in a more individualized fashion, improving overall patient care and eventually triaging patients to centers of excellence.

\section{References}

Bandiera E, Romani C, Specchia C, et al (2011). Serum human epididymis protein 4 and Risk for Ovarian Malignancy Algorithm as new diagnostic and prognostic tools for epithelial ovarian cancer management. Cancer Epidemiol Biomarkers Prev, 20, 2496-506.

Bast RC Jr, Badgwell D, Lu Z, et al (2005). New tumor markers: CA125 and beyond. Int J Gynecol Cancer, 15, 274-81.

Berek JS, Crum C, Friedlander M (2012). Cancer of the ovary, fallopian tube, and peritoneum. Int J Gynaecol Obstet, 119, 118-29.

Berry NB, Cho YM, Harrington MA, et al (2004). Transcriptional targeting in ovarian cancer cells using the human epididymis protein 4 promoter. Gynecol Oncol, 92, 896-904.

Buamah P (2000). Benign conditions associated with raised serum CA-125 concentration. J Surg Oncol, 75, 264-5.

Chung SH, Lee SY, Ju W, Kim SC (2013). Clinical efficacy of serum human epididymis protein 4 as a diagnostic biomarker of ovarian cancer: A pilot study. Obstet Gynecol Sci, 56, 234-41.

DeLong ER, DeLong DM, Clarke-Pearson DeL (1988). Comparing the areas under two or more correlated receiver operating characteristic curves: a nonparametric approach. Biometrics, 44, 837-45.

Duffy MJ, Bonfrer JM, Kulpa J, et al (2005). CA125 in ovarian cancer: European Group on Tumor Markers guidelines for clinical use. Int J Gynecol Cancer, 15, 679 -91.

Escudero JM, Auge JM, Filella X, et al (2011). Comparison of serum human epididymis protein 4 with cancer antigen 125 as a tumor marker in patients with malignant and nonmalignant diseases. Clin Chem, 57, 1534-44.

Ferlay J, Soerjomataram I, Ervik M, et al (2013). GLOBOCAN 2012 v1.0, Cancer Incidence and Mortality Worldwide: IARC CancerBase No. 11 [Internet]. Lyon, France: International Agency for Research on Cancer, Available from: http://globocan.iarc.fr, accessed on June 01, 2015.

Ferraro S, Braga F, Lanzoni M, et al (2013). Serum human epididymis protein 4 vs carbohydrate antigen 125 for ovarian cancer diagnosis: a systematic review. J Clin Pathol, 66, 273-81.

Freydanck MK, Laubender RP, Rack B, et al (2012). Two-marker combinations for preoperative discrimination of benign and malignant ovarian masses. Anticancer Res, 32, 2003-8.

Fuith LC, Daxenbichler G, Dapunt O (1987). CA 125 in the serum and tissue of patients with gynecological disease. Arch Gynecol Obstet, 241, 157-64.

Fujiwara H, Suzuki M, Takeshima N, et al (2015). Evaluation of human epididymis protein 4 (HE4) and Risk of Ovarian Malignancy Algorithm (ROMA) as diagnostic tools of type I and type II epithelial ovarian cancer in Japanese women. Tumour Biol, 36, 1045-53. 
Galgano MT, Hampton GM, Frierson HF Jr (2006). Comprehensive analysis of HE4 expression in normal and malignant human tissues. Mod Pathol, 19, 847-53.

Havrilesky LJ, Whitehead CM, Rubatt JM, et al (2008). Evaluation of biomarker panels for early stage ovarian cancer detection and monitoring for disease recurrence. Gynecol Oncol, 110, 374-82.

Hellström I, Raycraft J, Hayden-Ledbetter M, et al (2003). The HE4 (WFDC2) protein is a biomarker for ovarian carcinoma. Cancer Res, 63, 3695-700.

Hertlein L, Stieber P, Kirschenhofer A, et al (2012). Human epididymis protein 4 (HE4) in benign and malignant diseases. Clin Chem Lab Med, 50, 2181-8.

Holcomb K, Vucetic Z, Miller MC, Knapp RC (2011). Human epididymis protein 4 offers superior specificity in the differentiation of benign and malignant adnexal masses in premenopausal women. Am J Obstet Gynecol, 205, 358. 1-6.

Huhtinen K, Suvitie P, Hiissa J, et al (2009). Serum HE4 concentration differentiates malignant ovarian tumours from ovarian endometriotic cysts. Br J Cancer, 100, 1315-9.

Jacob F, Meier M, Caduff R, et al (2011). No benefit from combining HE4 and CA125 as ovarian tumor markers in a clinical setting. Gynecol Oncol, 121, 487-91.

Karlsen MA, Sandhu N, Høgdall C, et al (2012). Evaluation of HE4, CA125, risk of ovarian malignancy algorithm (ROMA) and risk of malignancyindex (RMI) as diagnostic tools of epithelial ovarian cancer in patients with a pelvic mass. Gynecol Oncol, 127, 379-83.

Kim YM, Whang DH, Park J, et al (2011). Evaluation of the accuracy of serum human epididymis protein 4 in combination with CA125 for detecting ovarian cancer: a prospective case-control study in a Korean population. Clin Chem Lab Med, 49, 527-34.

Kobayashi E, Ueda Y, Matsuzaki S, et al (2012). Biomarkers for screening, diagnosis, and monitoring of ovarian cancer. Cancer Epidemiol Biomarkers Prev, 21, 1902-12.

Lenhard M, Stieber P, Hertlein L, et al (2011). The diagnostic accuracy of two human epididymis protein 4 (HE4) testing systems in combination with CA125 in the differential diagnosis of ovarian masses. Clin Chem Lab Med, 49, 2081-8.

Li F, Tie R, Chang K, et al (2012). Does risk for ovarian malignancy algorithm excel human epididymis protein 4 and CA125 in predicting epithelial ovarian cancer: a meta-analysis. BMC Cancer, 12, 258.

Livak KJ, Schmittgen TD (2001). Analysis of relative gene expression data using real-time quantitative PCR and the 2(-Delta Delta C(T)) Method. Methods, 25, 402-8.

Medeiros LR, Rosa DD, da Rosa MI, Bozzetti MC (2009). Accuracy of CA 125 in the diagnosis of ovarian tumors: a quantitative systematic review. Eur J Obstet Gynecol Reprod Biol, 142, 99-105.

Meden H, Fattahi-Meibodi A (1998). CA 125 in benign gynecological conditions. Int J Biol Markers, 13, 231-7.

Miralles C, Orea M, España P, et al (2003). Cancer antigen 125 associated with multiple benign and malignant pathologies. Ann Surg Oncol, 10, 150-4.

Molina R, Auge JM, Escudero JM, et al (2008). Mucins CA 125, CA 19.9, CA 15.3 and TAG-72.3 as tumor markers in patients with lung cancer: comparison with CYFRA 21-1, CEA, SCC and NSE. Tumour Biol, 29, 371-80.

Molina R, Escudero JM, Augé JM, et al (2011). HE4 a novel tumour marker for ovarian cancer: comparison with CA 125 and ROMA algorithm in patients with gynaecological diseases. Tumour Biol, 32, 1087-95.

Molina R, Ojeda B, Filella X, et al (1992). A prospective study of tumor markers CA 125 and CA 19.9 in patients with epithelial ovarian carcinomas. Tumour Biol, 13, 278-86.

Montagnana M, Danese E, Ruzzenente O, et al (2011). The ROMA (risk of ovarian malignancy algorithm) for estimating the risk of epithelial ovarian cancer in women presenting with pelvic mass: is it really useful? Clin Chem Lab Med, 49, 521-5.

Montagnana M, Lippi G, Ruzzenente O, et al (2009). The utility of serum human epididymis protein 4 (HE4) in patients with a pelvic mass. J Clin Lab Anal, 23, 331-5.

Moore RG, Brown AK, Miller MC, et al (2008). The use of multiple novel tumor biomarkers for the detection of ovarian carcinoma in patients with a pelvic mass. Gynecol Oncol, 108, 402-8.

Moore RG, McMeekin DS, Brown AK, et al (2009). A novel multiple marker bioassay utilizing HE4 and CA125 for the prediction of ovarian cancer in patients with a pelvic mass. Gynecol Oncol, 112, 40-6.

Moore RG, Miller MC, Disilvestro P, et al (2011). Evaluation of the diagnostic accuracy of the risk of ovarian malignancy algorithm in women with a pelvic mass. Obstet Gynecol, 118, 280-8.

Moore RG, Jabre-Raughley M, Brown AK, et al (2010). Comparison of a novel multiple marker assay vs the Risk of Malignancy Index for the prediction of epithelial ovarian cancer in patients with a pelvic mass. Am J Obstet Gynecol, 203, 1-6.

Niloff JM, Knapp RC, Schaetzl E, Reynolds C, Bast RC Jr (1984). CA125 antigen levels in obstetric and gynecologic patients. Obstet Gynecol, 64, 703-7.

Nolen B, Velikokhatnaya L, Marrangoni A, et al (2010). Serum biomarker panels for the discrimination of benign from malignant cases in patients with an adnexal mass. Gynecol Oncol, 117, 440-55.

Ortiz-Muñoz B, Aznar-Oroval E, García García A, et al (2014). HE4, Ca125 and ROMA algorithm for differential diagnosis between benign gynaecological diseases and ovarian cancer. Tumour Biol, 35, 7249-58.

Park Y, Lee JH, Hong DJ, Lee EY, Kim HS (2011). Diagnostic performances of HE4 and CA125 for the detection of ovarian cancer from patients with various gynecologic and nongynecologic diseases. Clin Biochem, 44, 884-8.

Park Y, Kim Y, Lee EY, Lee JH, Kim HS (2012). Reference ranges for HE4 and CA125 in a large Asian population by automated assays anddiagnostic performances for ovarian cancer. Int J Cancer, 130, 1136-44.

Partheen K, Kristjansdottir B, Sundfeldt K (2011). Evaluation of ovarian cancer biomarkers HE4 and CA-125 in women presenting with a suspicious cystic ovarian mass. J Gynecol Oncol, 22, 244-52.

Rosen DG, Wang L, Atkinson JN, et al (2005). Potential markers that complement expression of CA125 in epithelial ovarian cancer. Gynecol Oncol, 99, 267-77.

Ruggeri G, Bandiera E, Zanotti L, et al (2011).HE4 and epithelial ovarian cancer: comparison and clinical evaluation of two immunoassays and a combination algorithm. Clin Chim Acta, 412, 1447-53.

Seibaek L, Petersen LK, Blaakaer J, Hounsgaard L (2011). Symptom interpretation and health care seeking in ovarian cancer. BMC Women's Health, 11, 31 .

Somigliana E, Viganò P, Tirelli AS, et al (2004). Use of the concomitant serum dosage of CA 125, CA 19-9 and interleukin-6 to detect the presence of endometriosis. Results from a series of reproductive age women undergoing laparoscopic surgery for benign gynecological conditions. Hum Reprod, 19, 1871-6.

Sturgeon CM, Duffy MJ, Stenman UH, et al (2008). National academy of clinical biochemistry laboratory medicine 
Tissue CA125 and HE4 Gene Expression Offers Superior Accuracy in Discriminating Benign from Malignant Pelvic Masses practice guidelines for use of tumor markers in testicular, prostate, colorectal, breast, and ovarian cancers. Clin Chem, $\mathbf{5 4}, 11-79$.

Su Z, Graybill WS, Zhu Y (2013). Detection and monitoring of ovarian cancer. Clin Chim Acta, 415, 341-5.

Trapé J, Filella X, Alsina-Donadeu M, et al (2011). Increased plasma concentrations of tumour markers in the absence of neoplasia. Clin Chem Lab Med, 49, 1605-20.

Van Gorp T, Cadron I, Despierre E, et al (2011). HE4 and CA125 as a diagnostic test in ovarian cancer: prospective validation of the risk of ovarian malignancy algorithm. Br J Cancer, 104, 863-70.

Wang J, Gao J, Yao H, et al (2014). Diagnostic accuracy of serum HE4, CA125 and ROMA in patients with ovarian cancer: a meta-analysis. Tumour Biol, 35, 6127-38.

Yang Z, Wei C, Luo Z, Li L (2013). Clinical value of serum human epididymis protein 4 assay in the diagnosis of ovarian cancer: a meta-analysis. Onco Targets Ther, 6, 957-66.

Yu S, Yang HJ, Xie SQ, Bao YX (2012). Diagnostic value of HE4 for ovarian cancer: a meta-analysis. Clin Chem Lab Med, 50, 1439-46.

Zhen S, Bian LH, Chang LL, Gao X (2014). Comparison of serum human epididymis protein 4 and carbohydrate antigen 125 as markers in ovarian cancer: A meta-analysis. Mol Clin Oncol, 2, 559-66.

Zheng H, Gao Y (2012). Serum HE4 as a useful biomarker in discriminating ovarian cancer from benign pelvic disease. Int J Gynecol Cancer, 22, 1000-5. 